\title{
On the Role of Avatars in Argumentation
}

\author{
Tom Blount \\ Web and Internet Science \\ Electronics and Computer \\ Science \\ University of Southampton \\ Southampton, United Kingdom \\ tb12g09@ecs.soton.ac.uk
}

\author{
David E. Millard \\ Web and Internet Science \\ Electronics and Computer \\ Science \\ University of Southampton \\ Southampton, United Kingdom \\ dem@ecs.soton.ac.uk
}

\author{
Mark J. Weal \\ Web and Internet Science \\ Electronics and Computer \\ Science \\ University of Southampton \\ Southampton, United Kingdom \\ mjw@ecs.soton.ac.uk
}

\begin{abstract}
The use of avatars in shared virtual spaces provides a useful vehicle with which people can present themselves in the way they choose. These avatars can also shape the behaviour of individuals, and their interactions with groups of users. Here, we make the case that there is scope for investigation into how avatars impact argumentation in virtual spaces, particularly how they alter the types of argumentation tactics that are used, and discuss some of the challenges that need to be addressed when researching this area.
\end{abstract}

\section{Categories and Subject Descriptors}

H.4.3 [Information Systems Applications]: Communications Applications-Social media

\section{Keywords}

argumentation; rhetoric; avatars; virtual spaces; social web

\section{INTRODUCTION}

Argumentation, discussion and rhetoric play an important part in shaping the interactions of users of the social web and, as a result, the development and expansion of the social web itself. Within argumentation in a social setting, rhetoric in particular (or the art of persuading an audience with knowledge of the topic at hand and, crucially, knowledge of the audience themselves) plays a valuable role.

Virtual social worlds (and virtual game worlds) are computer-simulated environments in which users can interact with one another and the environment itself [13]. These virtual spaces are a growing way in which people can meet both socially [12], recreationally [23] and even for business applications [7]. To facilitate these interactions, many virtual spaces provide the ability for a user to customise the appearance of their virtual self, or avatar. This ranges from selecting a name and profile picture as on a social network, to creating, customising and clothing a three-dimension representation of their choice which moves through and interacts with the virtual space. In this work we focus our discussions on the latter representations of avatar, due to the direct interaction this produces between avatars.

Permission to make digital or hard copies of all or part of this work for personal or classroom use is granted without fee provided that copies are not made or distributed for profit or commercial advantage and that copies bear this notice and the full citation on the first page. Copyrights for components of this work owned by others than ACM must be honored. Abstracting with credit is permitted. To copy otherwise, or republish, to post on servers or to redistribute to lists, requires prior specific permission and/or a fee. Request permissions from Permissions@acm.org.

NHT '15 September 01 2015, Guzelyurt, TRNC, Cyprus

(C) 2015 ACM. ISBN 978-1-4503-3797-7/15/09...\$15.00.

DOI: http://dx.doi.org/10.1145/2804565.2804569
Because of the scope of customisation options the user often has over the appearance of their avatar they are not limited to creating a likeness of themselves (although some choose to do so), but can choose to create whatever they feel best represents themselves, which goes on to shape the interactions and engagement that follows [24].

\section{USE OF AVATARS}

Social worlds, practically by definition, require an avatar for users to be able to interact in a meaningful manner. Second Life is an example of such a world, that allows users to visit seminars, attend concerts, go shopping or even visit historical eras such as ancient Rome [8]. One particularly noteworthy feature of Second Life is the right of users to maintain the copyrights of any content they create within the virtual world, which can then be sold to other users for virtual money, called Linden Dollars. These Linden Dollars can then be exchanged for real world currency, allowing users to, in essence, work a virtual job alongside their social activities [12].

Avatars are also used in a recreational or gaming context. World of Warcraft is a massively-multiplayer online role-playing game that allows players to create a character to explore and interact with a virtual world. Many of the races a player may chooses from are monstrous or fantastical, such as orcs, elves, or the minotaur-like tauren. There is, therefore, a limit to how closely a player can model their avatar after themselves (unless they restrict their race to human). The example of World of Warcraft also serves to highlight the notion of the avatar as a vehicle for play, versus the avatar as role itself. Some players use their avatar simply as a means to play the game, seeing it as a character to be controlled to further their experience, and hence their discussions revolve around the mechanical features of the game (such as their abilities and specialisations, or the statistics of items) [1]. Whereas, some players choose to roleplay, performing similar to an actor, as if their avatar was a real part of the virtual world, with goals, hopes and fears that may be very different from the controlling player's [18, 22].

Avatars are also used in areas other than social or recreational spaces. In industry, avatars and virtual spaces are used as a means of allowing employers, employees and collaborators to meet remotely, rather than resorting to a long and expensive commute [7]. In education, virtual worlds offer the potential for providing deeper engagement in online courses by allowing synchronous communication between students and teacher [19]. There have also been some efforts to tailor a virtual space for the purpose of conducting dialectic arguments and solving so-called "wicked" problems [15, 11]. Mention must also be made of the corporate interest in marketing within virtual worlds. Corporations maintain an active presence on 
popular social media platforms as a means of engaging with their users [13], and there is a growing interest in the platform of virtual worlds. While this interest currently focuses on the potential for advertising, such as erecting virtual billboards or hosting virtual events $[10,12]$, the inclusions of dedicated inhabitants in virtual worlds (overtly or otherwise) may well be the next logical step for directly engaging with, and persuading, current and potential customers.

\section{ARGUMENTATION TACTICS}

Argumentation is fundamental to human communication - it is how people share new information and new ideas, and propose courses of action that see them carried out $[9,17]$. As a result, there is a large amount of research on argumentation from a wide variety of disciplines and topics, including: philosophy, and the nature of fallacies and how they may be critically appraised [21]; sociology, and the need to differentiate between classical logic and social argumentation due to the need for the capability to reason using only partial knowledge [20]; law, and the need for measures of certainty and belief when modelling and reasoning over assertions [3]; and artificial intelligence, and the use of agent-based systems such as dialogue games, as methods for reasoning over argument $[2,14]$.

Central to many formal models of argument is the idea of logical reasoning: the principle that premises (that are assumed to be true) are advanced and used to draw conclusions, which may subsequently be attacked and defeated by newly formed premises. While this is an obvious choice to focus on, as it covers many features of argument as we understand it and is well suited towards modelling problem-solving (or dialectic) argumentation, it also neglects to model some of the techniques of social argumentation. Consider the analogy of two political opponents engaged in a hustings. Their arguments are not meant to convince the other of their position, or to join the "right" side. Instead, their arguments are for the benefit of the audience present. Therefore, attempts to "play the crowd", or endear oneself to the audience, should be equally important to the model in this context [4]. Other rhetorical techniques can include attempting to make oneself appear more intelligent, or more powerful than one's opponent in an attempt to have them back down (culminating at the extreme in outright threats in an attempt to silence them). In case studies, rhetorical tactics have been shown to be frequently used alongside logical tactics on the social web [5]. Presentation of self, therefore, is a key element of rhetorical tactics and argumentation on the social web itself.

\section{THE ROLE OF AVATAR IN ARGUMENTATION}

There are a number of distinct areas of research that can be pursued with regard to the role that avatars play in argumentation. Firstly, whether there is a need for encouraging, or providing a dedicated space for, dialectic argumentation in virtual worlds. Hall et al. take a direct approach, and present an environment within which participants can model an argument or discussion taking place on a virtual pinboard [11]; however, their efforts centre on the virtual space itself, and its use as an argumentation tool, rather than the avatars of the users inhabiting that space. They also discuss that technological approaches, such as virtual avatars, may prove ill-suited to directly facilitate collaborative argumentation, when compared with direct face-to-face communications.
There is also the question of whether it is preferable for avatars in such as space to represent their human counterparts, or whether a dialectic argumentation would be better facilitated by making all avatars equal (which arguably runs counter to the purpose of their inclusion).

This leads on to the second topic, the impact of avatars on the perception of the audience. Usernames alone can shape the way in which a user is perceived [6]; with the addition of an avatar this perception can be altered even further. Gender of the avatar has been shown to have a high impact on how it is received by the audience. Audiences are more likely to directly help a female avatar than a male avatar [16] and are also more easily persuaded by avatars of the opposite gender [26]. Further work in this area should explore the impact of additional features of avatars and the environment in which they are presented.

Finally, the previous topic can be inverted to place the focus on examining how the avatar affects the controller themselves. It has already been shown that users will behave differently based on the avatar they control. For example, users with attractive avatars are more confident and open than those with unattractive avatars, while users with shorter avatars are more submissive than those with taller avatars. This occurrence is known as the Proteus effect [24]. It is likely that the type of avatar a user controls also has an effect on the way in which they apply argumentation techniques, compared to how they might in other areas of the social web, or in person. Exactly what impact the Proteus effect has on the use of argumentation tactics remains to be seen. One of the interesting research areas involving the Proteus effect is how much of this behavioural adaptation then feeds back into the real world [25] which raises the possibility that so-called "toxic" behaviour in social worlds may lead to increased hostility in real world interactions. In addition to the notion of subconscious behaviour effects caused due to the relationship the user maintains with their avatar, there is the possibility that users may also consciously choose an avatar, or certain features of it, and adopt different behaviour in an attempt to exploit their audience to their own ends.

There are also a number of challenges to be aware of within this area. Perhaps the most obvious of these is the unique media richness and diversity of purpose of virtual worlds. Given the wide variety of different types of avatar and environment, from monstrous races in a fantasy setting to real-life fascicles of people in a corporate boardroom. Modelling all of these overlapping features is a challenge in itself, but it also leads to the next point for consideration: the purpose for which the user has created the avatar. The purpose of the virtual world, be it socialising, gaming, or debating, will directly affect not only how a user will design their avatar to appear, but also how the user sees their avatar, whether it is a facsimile of themselves, an extension of themselves or a "separate" entity altogether. For example, a user in one instance might argue about which item of virtual armour is best for their style of gameplay and in another argue "in character" about the history and philosophy of the virtual world they inhabit, with the use of different argumentation tactics for each. Similar restrictions are also present within virtual worlds both in terms of the creative freedom a user has to design their avatar (whether they can pick from pre-existing characters, make broad changes such as gender, race and clothing, or "fine tune" their avatar making changes such as eye colour, facial features and hairstyles) and in terms of, once again, the purpose of the virtual world. In World of Warcraft, for example, the role a player wishes to play (e.g. a magic user, a warrior, a hunter, etc.) 
has a bearing on which race they choose. The hulking tauren, for example, cannot play as the stealthy rogue class and hence the experience a player wishes to have restricts the type of avatar they can create. This notion of identity, when combined with the Proteus effect, may also lead to dramatically different argumentation strategies.

\section{CONCLUSIONS}

The use of argumentation, discussion and debate across the social web and within virtual worlds is unavoidable and, indeed, something that in many cases should be encouraged. Rhetorical tactics that form a key element of these debates revolve around the audience's perception of the participants. However, due to the nature of avatars, participants can shape or craft their appearance to suit their needs and purpose. While for the majority of cases on the social web this will not be specifically to win an argument, it is likely that this ability will have an impact on the course of debates in the virtual sphere. As the prevalence of the social web increases, it is of growing importance to fully understand the effects of the use of avatars on inter-personal communication, particularly with regard to argumentation.

\section{Acknowledgements}

The authors would like to thank Elzabi Rimington for giving direction towards particularly useful areas of literature on this topic.

\section{REFERENCES}

[1] E. Alagoz. Social argumentation in online synchronous communication. International Journal of Computer-Supported Collaborative Learning, 8(4):399-426, 2013.

[2] T. J. M. Bench-Capon and P. E. Dunne. Argumentation in artificial intelligence. Artificial intelligence, 171(10):619-641, 2007.

[3] S. Bertea. Certainty, reasonableness and argumentation in law. Argumentation, 18(4):465-478, 2004.

[4] T. Blount, D. E. Millard, and M. J. Weal. Towards Modelling Dialectic and Eristic Argumentation on the Social Web. In 14th workshop on Computational Models of Natural Argument, 2014.

[5] T. Blount, D. E. Millard, and M. J. Weal. An Investigation into the Use of Logical and Rhetorical Tactics within Eristic Argumentation on the Social Web. In ACM Conference on Hypertext and Social Media, 2015.

[6] K. M. Cornetto and K. L. Nowak. Utilizing usernames for sex categorization in computer-mediated communication: Examining perceptions and accuracy. CyberPsychology \& Behavior, 9(4):377-387, 2006.

[7] T. Erickson, N. S. Shami, W. A. Kellogg, and D. W. Levine. Synchronous interaction among hundreds: an evaluation of a conference in an avatar-based virtual environment. In Proceedings of the SIGCHI Conference on Human Factors in Computing Systems, pages 503-512. ACM, 2011.

[8] S. Gottschalk. The presentation of avatars in second life: Self and interaction in social virtual spaces. Symbolic interaction, 33(4):501-525, 2010.

[9] U. Hahn, M. Oaksford, and A. Corner. Circular arguments, begging the question and the formalization of argument strength. In Proceedings of AMKLC'05, International Symposium on Adaptive Models of Knowledge, Language and Cognition, pages 34-40, 2005.
[10] P. Hemp. Avatar-based marketing. Harvard business review, 84(6):48-57, 2006.

[11] M. John Hall, K. Jones, P. Bermell-Garcia, and D. David Hansen. Argumentation in virtual collaborative environments addressing complex issues through remote synchronous collaboration. In Systems Conference (SysCon), 2015 9th Annual IEEE International, pages 249-255. IEEE, 2015.

[12] A. M. Kaplan and M. Haenlein. The fairyland of second life: Virtual social worlds and how to use them. Business horizons, 52(6):563-572, 2009.

[13] A. M. Kaplan and M. Haenlein. Users of the world, unite! The challenges and opportunities of Social Media. Business horizons, 53(1):59-68, 2010.

[14] N. C. Karunatillake, N. R. Jennings, I. Rahwan, and P. McBurney. Formal Semantics of ABN Framework. Technical report, University of Southampton, 2008.

[15] W. Kunz and H. W. Rittel. Issues as elements of information systems, volume 131. Institute of Urban and Regional Development, University of California Berkeley, California, 1970.

[16] M. Lehdonvirta, Y. Nagashima, V. Lehdonvirta, and A. Baba. The stoic male how avatar gender affects help-seeking behavior in an online game. Games and Culture, 7(1):29-47, 2012.

[17] A. d. Moor and M. Aakhus. Argumentation support: From technologies to tools. Communications of the ACM, 49(3):93-98, Mar. 2006.

[18] T. E. Mortensen. Wow is the new mud social gaming from text to video. Games and Culture, 1(4):397-413, 2006.

[19] A. Petrakou. Interacting through avatars: Virtual worlds as a context for online education. Computers \& Education, 54(4):1020-1027, 2010.

[20] L. Pólos and M. T. Hannan. Reasoning with partial knowledge. Sociological methodology, 32(1):133-181, 2002.

[21] C. W. Tindale. Fallacies and argument appraisal. Cambridge University Press, 2007.

[22] D. Williams, N. Ducheneaut, L. Xiong, Y. Zhang, N. Yee, and E. Nickell. From tree house to barracks the social life of guilds in world of warcraft. Games and culture, 1(4):338-361, 2006.

[23] J. Wu, P. Li, and S. Rao. Why they enjoy virtual game worlds? an empirical investigation. Journal of Electronic Commerce Research, 9(3):219-230, 2008.

[24] N. Yee and J. Bailenson. The proteus effect: The effect of transformed self-representation on behavior. Human communication research, 33(3):271-290, 2007.

[25] N. Yee, J. N. Bailenson, and N. Ducheneaut. The proteus effect: Implications of transformed digital self-representation on online and offline behavior. Communication Research, 36(2):285-312, 2009.

[26] C. Zanbaka, P. Goolkasian, and L. Hodges. Can a virtual cat persuade you?: the role of gender and realism in speaker persuasiveness. In Proceedings of the SIGCHI conference on Human Factors in computing systems, pages 1153-1162. ACM, 2006. 